Iranian Journal of Pathology | ISSN: 2345-3656

\title{
Annexin Expression in Cholangiocarcinoma, and Metastatic Pancreatic Ductal Adenocarcinoma "Is it be Helpful for Differential Diagnosis of These Tumors in the Liver?"
}

\author{
Bita Geramizadeh $^{1,2}$ D, Mahsa Sehat ${ }^{1}$, Azam Mehrmozayan ${ }^{1}$, Ali Reza Shojazadeh ${ }^{1}$ \\ 1. Department of Pathology, School of Medicine, Shiraz University of Medical Sciences, Shiraz, Iran \\ 2. Transplant Research Center, Shiraz University of Medical Sciences, Shiraz, Iran
}

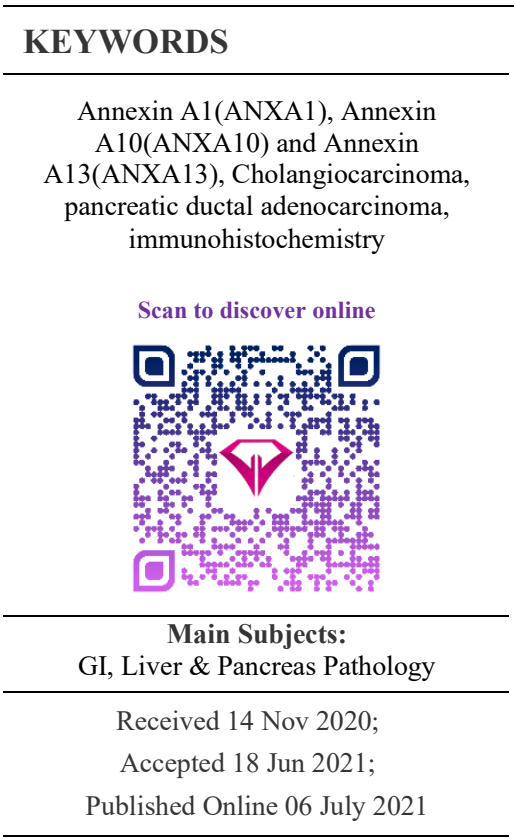

10.30699/IJP.20201.138489.2512

\section{ABSTRACT}

Background \& Objective: Differential diagnosis between cholangiocarcinoma (CCA) and metastatic pancreatic ductal adenocarcinoma (PDA) in the liver is difficult and so far, no specific immunohistochemical marker is reported to differentiate these two tumors. Considering the existing literature, the level of expression of Annexins (Annexin A1, 10 and 13) have been studied for differential diagnosis between these two tumors by molecular methods and promising results have been reported. Therefore, in this study, we tried to investigate the immunohistochemical value of these three Annexins for the differential diagnosis of CCA and PDA in the liver.

Methods: The articles that reported the research subject in 10 years (2009-2019), including 45 cases of CCA and 50 cases of metastatic PDA in the liver were evaluated considering the presence or absence of AnnexinA1 (ANXA1), Annexin A10 (ANXA10) and Annexin A13 (ANXA13) expression by immunohistochemistry, were investigated.

Results \& Conclusion: This study showed, ANXA1 was positive both in PDA and CCA, ANXA10 was positive in $\sim 60 \%$ of PDA cases and $\sim 40 \%$ of CCA cases, and ANXA13 was mostly negative in both groups. The best sensitivity was found in cytoplasmic and nuclear ANXA1 (80\% and 84\%, respectively) to distinguish PDA from CCA and vice versa. The best specificity was observed in ANXA10 and ANXA13 to distinguish PDA from CCA. Also, ANXA13 had the best specificity to distinguish CCA from PDA. Our investigations showed that, ANXA1 probably can classify positive cases correctly, but it cannot discriminate PDA from CCA. ANXA10 had fair sensitivity and specificity to discriminate PDA from CCA. ANXA13 apparently had a high specificity that can help to narrow-down the differential diagnoses.

Copyright (C) 2021. This is an open-access article distributed under the terms of the Creative Commons Attribution- 4.0 International License which permits Share, copy and redistribution of the material in any medium or format or adapt, remix, transform, and build upon the material for any purpose, even commercially.

\section{Introduction}

Malignant liver tumors are either primary or metastatic. Most common primary liver tumors in adults are hepatocellular carcinoma (HCC) and cholangiocarcinoma (CC). Histopathology of CC is very similar to pancreatic ductal adenocarcinoma (PDA) and so far, there has been no immunohistochemical marker to differentiate pancreatic ductal adenocarcinoma and cholangiocarcinoma (1).

Annexins (ANX) are calcium-dependent phospholipid-binding proteins comprising 13 members in human (ANXA1-A11, A13 and A8L1; A12 is unassigned). Annexins are involved in different types of biological processes such as anti-inflammation, cell differentiation, apoptosis, and proliferation. Aberrant expression of Annexins have been reported in different cancers as tumor promoters and suppressors, but the results in liver cancers are controversial and the level of their expression and usefulness in differential diagnosis in different types of liver malignancies is unclear (2).

In this study we tried to use Annexins in $\mathrm{CC}$ and PDA in the documented cases to find out if these markers can be helpful for differential diagnosis of these two tumors in the liver.

\section{Patients and Methods}

Studying the literature between 2009 and 2019, 95 cases were selected with the definite diagnosis of intrahepatic CCA and metastatic PDAC to the liver in Whipple's operation specimens from the archives of pathology department of affiliated hospitals of Shiraz University of Medical Sciences, Shiraz, Iran. We 
selected 45 cases of $\mathrm{CC}$ and 50 cases of PDA with definite diagnosis and enough tissue and good quality (no necrosis and proper fixation). Cases with unproven diagnosis or inadequate tissue or extensive necrosis have been excluded from the study.

The slides were reviewed, and the best representative blocks were selected to be stained with annexins.

The detailed characteristics of each of the antibodies are shown in Table 1.

All the immunohistochemical slides were reviewed by a hepatopathologist and a general pathologist, both of whom were blinded to the final diagnosis (Gold standard). Figures 1, 2, $\underline{3}$ and $\underline{4}$ are sample pictures from our cases.

Statistical analysis was carried out using statistical package for social sciences (SPSS) Version 22.0. (IBM Corp., Armonk, NY, USA). Qualitative and quantitative variables were described using frequency (percent) and mean \pm Standard Deviation (SD), and visualized by box plot. Different variables were compared among the two groups using the Chi-square test for qualitative variables and the Independent t-test for quantitative variables. Also, contingency table was used for obtaining diagnostic test statistics. P-value $\leq$ 0.05 was considered statistically significant.

Table 1. Characteristics of Annexin Antibodies which have been used to differentiate CC and PDA in the liver (ANXA1, ANXA10, and ANXA13)

\begin{tabular}{|c|c|c|c|c|c|}
\hline Antibody & $\begin{array}{l}\text { Antigen } \\
\text { Retrieval }\end{array}$ & Lot Number & Description & Brand & Positive control \\
\hline ANX A1 & TE Buffer & 04250009 & Polyclonal/Rabbit & Invitrogen & $\begin{array}{l}\text { Bone } \\
\text { marrow/hairy } \\
\text { leukemia }\end{array}$ \\
\hline ANX A10 & TE Buffer & 4 H2835076A & Polyclonal/Rabbit & Invitrogen & Gastric mucosa \\
\hline ANX A13 & TE Buffer & RO8000 & Polyclonal/Rabbit & Invitrogen & Duodenal mucosa \\
\hline
\end{tabular}

\section{Results \& Discussion}

A group of 45 patient with CCA and a group of 50patient with PDAC were qualified for analysis (according to inclusion criteria). Sex distribution was not statistically different $(P=0.12)$ but age was significantly higher in PDAC group $(P=0.008)$. Demographic and tumoral variables of two groups are depicted and compared in Table 2.

As shown in Table 3, ANXA10 was significantly more intensely positive in CCA group comparing with

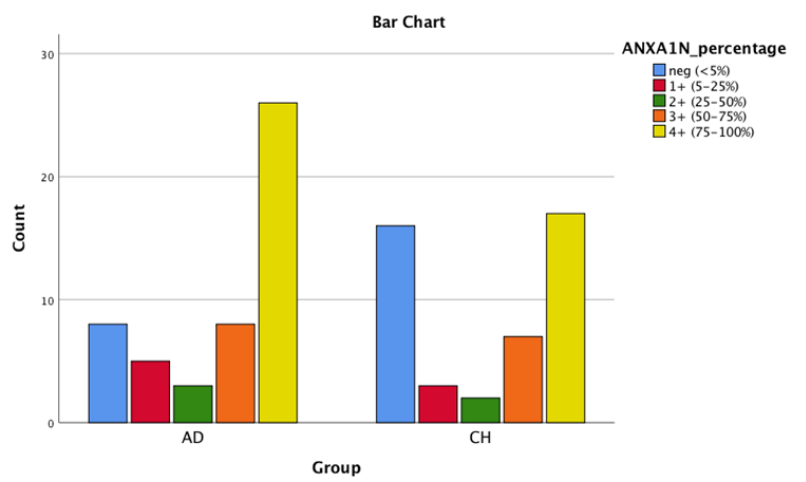

Fig. 1. Bar chart shows the comparison in Annexin1 nuclear staining in PDA and CCA.
PDAC group $(P$ (cytoplasmic) $=0.023 ; P$ (nuclear)$=0.035$ ). It should be mentioned that ANXA1 tends to be positive in both groups, ANXA10 stained in $\sim 60 \%$ of PDAC cases and $\sim 40 \%$ of CCA cases, and ANXA13 was mostly negative in both groups. Figures $\underline{1}, \underline{2}, \underline{3}, \underline{4}, \underline{5}$ and $\underline{6}$ depict bar chart comparison of IHC staining percentage result of annexins in PDAC and CCA.

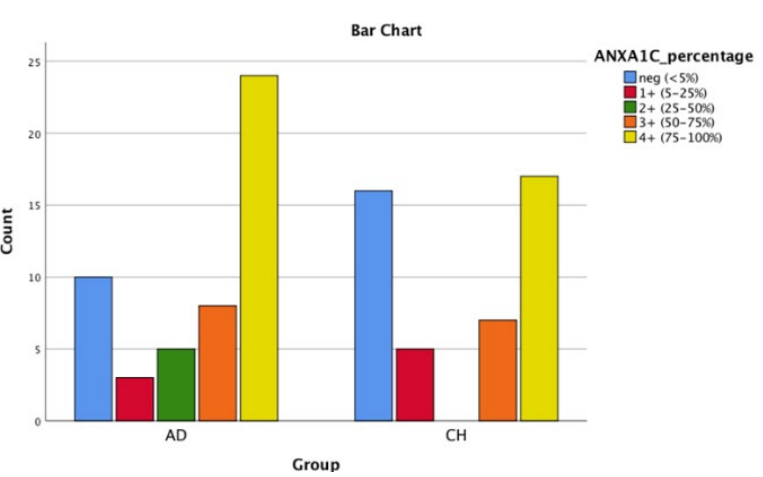

Fig. 2. Bar chart shows AnnexinA1 cytoplasmic positivity in PDA and CCA. 
The best sensitivity was related to cytoplasmic and nuclear ANXA1 (80\% and $84 \%$, respectively) to distinguish PDAC from CCA and vice versa (Tables 4 and ).

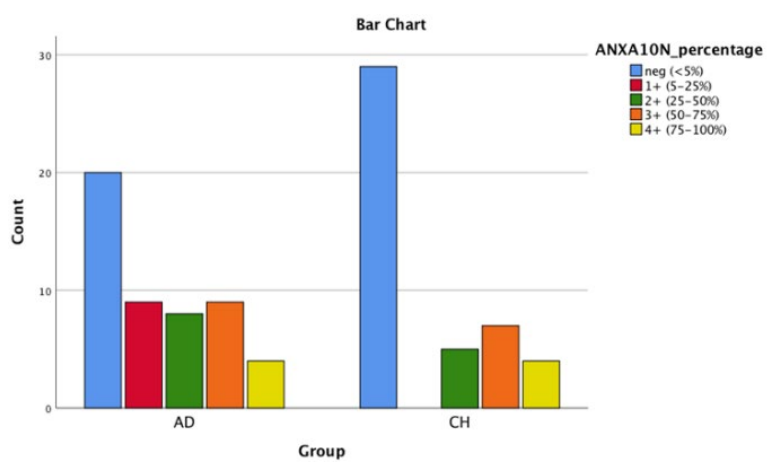

Fig. 3. Bar chart shows AnnexinA10 nuclear positivity in PDA and CCA.

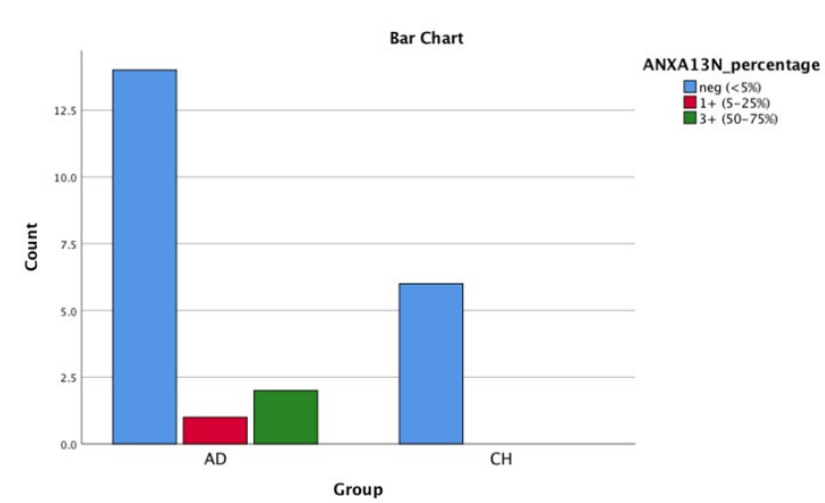

Fig. 5. Bar chart shows AnnexinA13 nuclear positivity in PDA and CCA.
The best specificity was observed in ANXA10 and ANX13 to distinguish PDAC from CCA (Table 4). Also, ANXA13 had the best specificity to distinguish CCA from PDAC (Table 5).

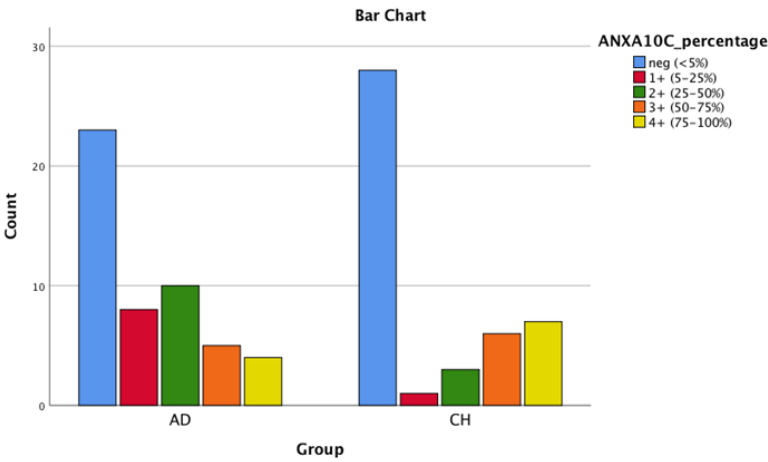

Fig. 4. Bar chart shows AnnexinA10 cytoplasmic positivity in PDA and CCA.

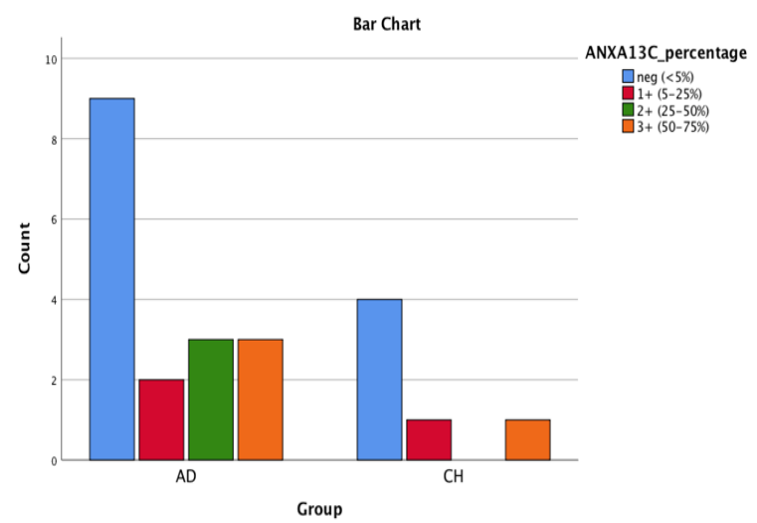

Fig. 6. Bar chart shows AnnexinA13 cytoplasmic positivity in PDA and CCA

Table 3. IHC results and comparison in the cases of CCA and PDA (N=Nuclear, $\mathrm{C}=$ Cytoplasmic)

\begin{tabular}{|c|c|c|c|c|c|c|c|c|c|c|c|}
\hline & \multicolumn{5}{|c|}{$\operatorname{PDAC}(\mathrm{n}=50)^{1}$} & \multicolumn{5}{|c|}{$\operatorname{CCA}(n=45)^{1}$} & $\begin{array}{c}\text { P- } \\
\text { value }^{2}\end{array}$ \\
\hline & neg. & $1+$ & $2+$ & $3+$ & $4+$ & neg. & $1+$ & $2+$ & $3+$ & $4+$ & \\
\hline ANXA1(N) & $16 \%$ & $10 \%$ & $6 \%$ & $16 \%$ & $52 \%$ & $35.6 \%$ & $6.7 \%$ & $4.4 \%$ & $15.6 \%$ & $37.8 \%$ & 0.28 \\
\hline $\operatorname{ANXA1}(C)$ & $20 \%$ & $6 \%$ & $10 \%$ & $16 \%$ & $48 \%$ & $35.6 \%$ & $11.1 \%$ & 0 & $15.6 \%$ & $37.8 \%$ & 0.095 \\
\hline $\operatorname{ANXA10}(\mathbf{N})$ & $40 \%$ & $18 \%$ & $16 \%$ & $18 \%$ & $8 \%$ & $64.4 \%$ & 0 & $11.1 \%$ & $15.6 \%$ & $8.9 \%$ & 0.023 \\
\hline $\operatorname{ANXA10}(C)$ & $46 \%$ & $16 \%$ & $20 \%$ & $10 \%$ & $8 \%$ & $62.2 \%$ & $2.2 \%$ & $6.7 \%$ & $13.3 \%$ & $15.6 \%$ & 0.035 \\
\hline ANXA13(N) & $82.4 \%$ & $5.9 \%$ & 0 & $11.8 \%$ & 0 & $100 \%$ & 0 & 0 & 0 & 0 & 0.544 \\
\hline ANXA13 (C) & $52.9 \%$ & $11.8 \%$ & $17.6 \%$ & $17.6 \%$ & 0 & $66.7 \%$ & $16.7 \%$ & 0 & $16.7 \%$ & 0 & 0.731 \\
\hline
\end{tabular}

Abbreviations: (1) PDAC: Pancreatic ductal adenocarcinoma, (2) CCA: Cholangiocarcinoma, (3) IHC: immunohistochemistry, (4) neg. $[<5 \%] ; 1+[5-25 \%] ; 2+[25-50 \%] ; 3+[50-75 \%] ; 4+[75-100 \%] .{ }^{1}$ due to resource limitation, sample size for cytoplasmic and nuclear ANXA13 testing was 17 and 6 for PDAC and CCA, respectively, ${ }^{2}$ Chi-square test. 
Table 4. Diagnostic test results (sensitivity, specificity, PPV and NPP) of ANXAs to distinguish PDAC from CCA (N, Nuclear \& C, Cytoplasmic)

\begin{tabular}{|c|c|c|c|c|}
\hline & Sensitivity & Specificity & PPV & NPV \\
\hline ANXA1(N) & $84 \%$ & $35.6 \%$ & $59.2 \%$ & $66.7 \%$ \\
\hline ANXA1(C) & $80 \%$ & $35.6 \%$ & $58 \%$ & $61.5 \%$ \\
\hline ANXA10(N) & $60 \%$ & $64.4 \%$ & $65.2 \%$ & $59.2 \%$ \\
\hline ANXA10 (C) & $54 \%$ & $62.2 \%$ & $61.4 \%$ & $54.9 \%$ \\
\hline ANXA13(N) & $17.6 \%$ & $100 \%$ & $100 \%$ & $30 \%$ \\
\hline ANXA13(C) & $47.1 \%$ & $66.7 \%$ & $80 \%$ & $30.8 \%$ \\
\hline
\end{tabular}

Abbreviations: (1) PPV: positive predictive value, (2) NPV: negative predictive value, (3) PDAC: Pancreatic ducta adenocarcinoma, (4) CCA: Cholangiocarcinoma.

Table 5. Diagnostic test results (sensitivity, specificity, PPV and NPP) of ANXAs to distinguish CCA from PDAC

\begin{tabular}{|c|c|c|c|c|}
\hline & Sensitivity & Specificity & PPV & NPV \\
\hline Nuclear ANXA1 & $64.4 \%$ & $16 \%$ & $40.8 \%$ & $33.3 \%$ \\
\hline Cytoplasmic ANXA1 & $64.4 \%$ & $20 \%$ & $42 \%$ & $38.5 \%$ \\
\hline Nuclear ANXA10 & $35.6 \%$ & $40 \%$ & $34.8 \%$ & $40.8 \%$ \\
\hline Cytoplasmic ANXA10 & $37.8 \%$ & $46 \%$ & $38.6 \%$ & $45.1 \%$ \\
\hline Nuclear ANXA13 & $0 \%$ & $82.4 \%$ & $0 \%$ & $70 \%$ \\
\hline Cytoplasmic ANXA13 & $33.3 \%$ & $52.9 \%$ & $20 \%$ & $69.2 \%$ \\
\hline
\end{tabular}

Abbreviations: (1) PPV: positive predictive value, (2) NPV: negative predictive value, (3) PDAC: Pancreatic ductal adenocarcinoma, (4) CCA: Cholangiocarcinoma.

One of the common challenges in the pathology of liver tumors is the differential diagnosis between CCA and metastatic PDA. So far there has not been any useful immunohistochemical marker to differentiate these two tumors especially in small liver biopsies. There are very few studies in the literature with proven useful biomarkers for this differential diagnosis. We considered Padden et al. study regarding the usefulness of Annexin A1, 10 and 13 by proteomics to differentiate CCA in the liver from metastatic PDA to the liver (3). So, we tried to use the same markers by immunohistochemistry on the tissues of the liver tumors to find out their role in differential diagnosis of hepatic CC and liver metastasis of PDA.

-Annexin A1: In the previous report, it has been shown that ANXA1 is linked to tumor development and progression $(4,5,6)$. It has been shown that that ANXA1 downregulated in esophageal, gastric, and CC and upregulated in PDA (7).

Wang et al. (2010) studied the expression of ANXA1 amongst 61 cases of hilar cholangiocarcinoma. They showed that ANXA1 was not expressed in $55.7 \%$ of cases and its downregulation was linked to lymph node metastases, histopathologic grade, recurrence, and poor prognosis (8). In a study on 68 , in 2013 Hongsrichan et al. (2) showed that ANXA1 was expressed in more than $90 \%$ of patients with CC.
Our results for CC was different with Wang et al. (2010) (8) study and similar to Hongsrichan et al. (2013) (2) study. We showed that more than $60 \%$ of cases with confirmed diagnosis of CC were positive for ANXA1. However, in Hongsrichan's study all samples were associated to an Opisthorchis viverrini infection, which might affect the protein expression profile of the cancerous cells. None of our cases showed this association (2).

Liu et al. (2016) studied ANXA1 expression in 162 cases of PDA. They showed that decreased expression of ANXA1 is associated with poor differentiation, lymph node metastasis, advanced TNM stage and poor survival of PDAC. These findings were again supported by ANXA1 gene knockdown assessment, which inhibited cell proliferation through G1 phase cell cycle arrest and by modulating MMP-9 activity and its inhibitor TIMP-1 significantly increased PDAC cell migration and invasion (6). In contrast, Gao et al. (2014) showed that ANXA1 was overexpressed in PDA. However, their sample size was very small (4 patients) (7). In another study by Bai et al. (2004) reported that ANXA1 overexpressed in PDA compare to its normal adjacent tissue (9). Padden et al. compared 73 patients with cholangiocarcinoma and 96 patients with PDAC (primary: $n=78$, metastatic: $n=18$ ) regarding ANXA1, 10 and 13 expression (3). Their results were similar to the above-mentioned studies (79), i.e. ANXA1 was overexpressed in PDA. They 
concluded that ANXA1 is a reliable biomarker for differential diagnosis between PDA from $\mathrm{CC}$ by a sensitivity of $84 \%$ and a specificity of $85 \%$ (3).

In our study regarding PDA, we showed that $\sim 80 \%$ of the cases with confirmed diagnosis of PDA express ANXA1, which was similar to the results of Gao et al. (2014) (7), Bai et al. (2004) (9) and Padden et al. (2015) (3). Similar to Padden's study, we showed that ANXA1 had the best sensitivity to discriminate PDA from CCA and vice versa; but, in our study, the specificity was low. This means that ANXA1 probably can classify positive cases correctly, but it cannot discriminate PDA from CCA.

ANXA10: This marker is reported to be overexpressed in PDA. In some reports, ANXA10 had a sensitivity of $90 \%$ and specificity of $66 \%$ in discriminating PDA from cholangiocarcinoma (3). Similarly, in the study by Liu et al. (2013), 78\% of primary PDA tumors, $83 \%$ of metastatic PDA tumors and only $17 \%$ of cholangiocarcinomas showed positive ANXA10 staining. ANXA10 has reported as marker with an excellent specificity but relatively low sensitivity for tumors with pancreatobiliary origin (6).

Kälsch et al. (2017), tested various markers for the discrimination between intrahepatic cholangiocarcinoma and metastases of PDA, comprising Annexin A1, Annexin A10, MUC5 AC, CK17, and N-Cadherin. They showed that ANXA10 was positive in $25 \%$ of CCA cases and $85 \%$ of the cases with the confirmed diagnosis of PDA. The authors recommended ANXA10 as the best immunohistochemical biomarker to discriminate between CCA and PDA (1).

Our results were different and expressed that ANXA10 was positive in $\sim 60 \%$ of the cases with the diagnosis of PDA and $\sim 40 \%$ of the cases of CCA. The sensitivity and specificity of ANNA10 to discriminate PDA from CCA was $\sim 60 \%$ and $\sim 65 \%$, respectively. So, our study did not confirm ANXA10 as the best biomarker to distinguish PDA vs. CCA.

ANXA13: There are quite a few studies about ANXA1 and ANXA10, but to the best of our knowledge there is only one study in field of PDA and
ANXA13. In the study by Padden et al. higher expression of ANXA13 was found in CCA being compared with PDA with a sensitivity of $84 \%$ and a specificity of 55\%. ANXA13 has been positive in 8 out of 18 PDA cases; therefore, ANXA13 was not a good biomarker to distinguish CCA from PDAC. (10)

In our study, the majority of the cases in both groups were negative for ANNXA13. However, it didn't have a good sensitivity to distinguish CCA from PDA and vice versa, but its high specificity can be helpful to narrow-down the differential diagnoses.

\section{Conclusion}

In summary, ANXA1 had the best sensitivity to detect PDA and CCA, but the specificity was low. Therefore, ANXA1 probably can classify positive cases correctly, but it cannot discriminate PDA from CCA to narrow-down the list of differential diagnoses. ANXA10 had fair sensitivity and specificity to discriminate PDA from CCA. So, we cannot represent ANXA10 as the best biomarker to distinguish PDA vs. CCA. ANXA13 apparently had a high specificity that can help to narrow-down the differential diagnoses.

The results of our study showed that ANXA1, A10 and A13 can be considered as good immunohistochemical biomarker candidates to differentiate CCA and PDA, however they should be interpreted in a panel in conjunction with the clinical and imaging findings.

\section{Acknowledgements}

This study is supported by deputy dean of school of medicine based on research projects number 12992-0101-95.

\section{Conflict of Interest}

The authors declared no conflict of interest.

\section{References}

1. Kälsch J., et al. Annexin A10 optimally differentiates between intrahepatic cholangiocarcinoma and hepatic metastases of pancreatic ductal adenocarcinoma: a comparative study of immunohistochemical markers and panels, Virchows Arch. 2017 ;470(5):537-543. [DOI:10.1007/s00428-017-2114-2]

2. Hongsrichan N, Rucksaken R, Chamgramol Y, Pinlaor P, Techasen A, Yongvanit P, Khuntikeo N, Pairojkul C, Pinlaor S, Annexin A1: A new immunohistological marker of cholangiocarcinoma, World J Gastroenterol. 2013 ;19(16):2456-65. [DOI:10.3748/wig.v19.i16.2456]
3. Padden J, Ahrens M, Kalsch J, Bertram S, Megger DA, Bracht $\mathrm{T}$, et al. Immunohistochemical markers distinguishing Cholangiocellular carcinoma (CCC) from pancreatic ductal adenocarcinoma (PDAC) discovered by proteomic analysis of microdissected cells. Mol Cell Proteomics. 2016;15:1072-1082. [DOI:10.1074/mcp.M115.054585]

4. Kang JS, Calvo BF, Maygarden SJ, Caskey LS, Mohler JL, Ornstein DK. Dysregulation of annexin I protein expression in high-grade prostatic intraepithelial 
neoplasia and prostate cancer. Clin Cancer Res. 2002;8(1):117-23.

5. Fernandez Moro C, Fernandez-Woodbridge A, Alistair D'souza M, Zhang Q, Bozoky B, Kandaswamy SV, et al. Immunohistochemical typing of adenocarcinomas of the pancreatobiliary system improves diagnosis and prognostic stratification. PLoS ONE. 2016;11(11): e0166067. [DOI:10.1371/journal.pone.0166067]

6. Liu QH, Shi ML, Bai J, Zheng JN., Identification of ANXA1 as a lymphatic metastasis and poor prognostic factor in pancreatic ductal adenocarcinoma, Asian Pac J Cancer Prev. 2015;16(7):2719-24. [DOI:10.7314/APJCP.2015.16.7.2719]

7. Gao Y, Chen Y, Xu D, Wang J, Yu G. Differential expression of ANXA1 in benign human gastrointestinal tissues and cancers. BMC Cancer. 2014;14:520 . [DOI:10.1186/1471-2407-14-520]
8. Wang D, Zhang H, Fang Z, Yu G., Annexin-1 downregulation is associated with clinical outcome in Chinese patients with hilar cholangiocarcinoma, Eur Surg Res. 2010;45(3-4):151-7. [DOI:10.1159/000320237]

9. Bai XF, Ni XG, Zhao P, Liu SM, Wang HX, Guo B, et al. Overexpression of annexin 1 in pancreatic cancer and its clinical significance. World J Gastroenterol. 2004;10(10):1466-70.

[DOI:10.3748/wig.v10.i10.1466]

10. Iglesias JM, Morgan RO, Jenkins NA, Copeland NG, Gilbert DJ, Fernandez MP., Comparative genetics and evolution of annexin A13 as the founder gene of vertebrate annexins, Mol Biol Evol. 2002 May;19(5):608-18 [DOI:10.1093/oxfordjournals.molbev.a004120] 